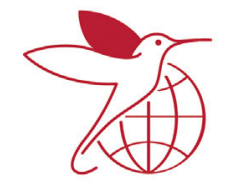

International

Diabetes

Federation

\title{
Prevalence of depression and its associations with cardio-metabolic control in Aboriginal and Anglo-Celt patients with type 2 diabetes: The Fremantle Diabetes Study Phase II
}

\author{
Timothy M.E. Davis ${ }^{a, *}$, Kerry Hunt ${ }^{a}$, David G. Bruce ${ }^{a}$, Sergio Starkstein ${ }^{b}$, \\ Timothy Skinner $^{c}$, Daniel McAullay ${ }^{d, e}$, Wendy A. Davis ${ }^{a}$ \\ ${ }^{a}$ University of Western Australia, School of Medicine \& Pharmacology, Fremantle Hospital, Fremantle, Western \\ Australia, Australia \\ ${ }^{\mathrm{b}}$ University of Western Australia, School of Psychiatry and Clinical Neurosciences, Fremantle Hospital, Fremantle, \\ Western Australia, Australia \\ c Charles Darwin University, School of Psychological and Clinical Sciences, Casuarina, Northern Territory, Australia \\ ${ }^{\mathrm{d}}$ Edith Cowan University, Kurongkurl Katitjin Centre for Indigenous Australian Education and Research, Mt Lawley, \\ Western Australia, Australia \\ ${ }^{\mathrm{e}}$ Australian National University, Australian Primary Health Care Research Institute, Canberra, Australian Capital \\ Territory, Australia
}

\section{A R T I C L E I N F O}

\section{Article history:}

Received 28 April 2014

Received in revised form

14 October 2014

Accepted 26 December 2014

Available online 21 January 2015

Keywords:

Type 2 diabetes

Depression

Aboriginal

Antidepressant treatment

Metabolic control

\begin{abstract}
A B S T R A C T
Aims: To determine the prevalence and associates of depression in Aboriginal and AngloCelt (AC) Australians with type 2 diabetes.

Methods: Community-based patients were screened using the Patient Health Questionnaire (PHQ-9) as part of detailed assessment. The prevalence of any current depression, major depression and antidepressant use by racial group was compared after adjustment for age, sex, educational attainment and marital status. Multiple logistic regression was used to determine associates of current depression.

Results: The 107 Aboriginal participants were younger (mean \pm SD $54.3 \pm 11.8$ vs $67.2 \pm 10.6$ years), less often male (34.6\% vs $50.9 \%$ ) and married (39.3\% vs $61.7 \%$ ), and more likely to smoke $(44.6 \%$ vs $8.1 \%)$ than the 793 AC subjects $(P \leq 0.002)$. Fifty-two Aboriginal $(48.5 \%)$ and 772 AC participants (97.4\%) completed the PHQ-9; these Aboriginals had similar sociodemographic, anthropometric and diabetes-related characteristics to those without PHQ9 data. A quarter of the Aboriginals had current depression vs $10.6 \%$ of ACs $(P=0.16), 15.4 \%$ vs $4.1 \%$ had major depression $(P=0.029)$, and $68.8 \%$ vs $29.7 \%$ had untreated depression $(P=0.032)$. Compared with non-depressed participants, patients with current depression were younger and more likely to smoke, to be overweight/obese and to have worse glycaemic control $(P \leq 0.024)$. Significant independent associates of current depression were educational attainment (inversely), smoking status, body mass index and fasting plasma glucose in the AC group and alcohol use in the Aboriginal group.
\end{abstract}

\footnotetext{
* Corresponding author at: University of Western Australia, School of Medicine and Pharmacology, Fremantle Hospital, PO Box 480, Fremantle, Western Australia 6959, Australia. Tel.: +61 894313229; fax: +61 894312977.

E-mail address: tim.davis@uwa.edu.au (Timothy M.E. Davis).

http://dx.doi.org/10.1016/j.diabres.2014.12.014

0168-8227/@ 2015 Elsevier Ireland Ltd. All rights reserved.
} 
Conclusions: Although prevalence of depression was not significantly increased in the Aboriginal patients, it was more likely to be major and untreated. Depression complicating type 2 diabetes is associated with adverse cardiovascular risk.

(C) 2015 Elsevier Ireland Ltd. All rights reserved.

\section{Introduction}

Diabetes and depression have a bi-directional relationship, with depressive disorders increasing the risk of type 2 diabetes $[1,2]$ and diabetes increasing the incidence of depression [3]. Depression has an adverse impact on glycaemic control and quality of life in patients with diabetes, and increases the risk of chronic complications and premature death [4-7]. Although these effects have been found in studies carried out in diverse populations, there are relatively few data on the prevalence and consequences of depression in indigenous patients with diabetes.

Individuals from minority and Aboriginal groups are likely to experience high levels of mental distress including depression [8]. However, in contrast to studies in largely Europid populations [3], it is possible that diabetes may not worsen this situation. A Canadian study found no differences in depressive symptoms between Aboriginal patients with diabetes or impaired glucose tolerance and normoglycaemic community members [9]. Notwithstanding significant issues with ascertainment, available national data suggest that Aboriginal Australians have double the risk of depression compared with non-Aboriginal individuals [10], but clinical audits have not always shown increased rates of diagnosed depression in those with diabetes. In one study carried out in 62 indigenous community health centres, the overall prevalence of diagnosed depression in people with diabetes was relatively low at $8.8 \%$ with a between-centre range of $0-37 \%$ [11], while in a related clinical audit of 44 health centres [12], $6 \%$ had documented depression (range 0-33\%). In addition, $18 \%$ of a sample of the patients with diabetes in this latter study had at least high-level mental distress on the Kessler Psychological Distress Scale (K-5) [12], compared with $31 \%$ in the general Aboriginal population [10].

There have been no systematic comparative studies of the prevalence of depression in Aboriginal people with diabetes, and none that have examined the influence of depression on self-care behaviours, cardiovascular risk factors and clinical management in this group. The aim of the present study was, therefore, to utilise baseline data from the Fremantle Diabetes Study (FDS) Phase II to determine whether there are differences in the frequency and associates of depression in Aboriginal compared with Anglo-Celt (AC) Australians with type 2 diabetes.

\section{Patients and methods}

\subsection{Patients}

The FDS Phase II is a longitudinal observational study carried out in a post-code-defined geographical area surrounding the port city of Fremantle in the state of Western Australia (WA). The study was approved by the Human Research Ethics Committee of the Southern Metropolitan Area Health Service and all patients gave written informed consent. Details of sample characteristics including classification of diabetes type and non-recruited patients have been published elsewhere [13]. In brief, any patient resident in the study catchment area with a clinician-verified diagnosis of diabetes was eligible. Sources of identification and/or diagnostic data included public hospital inpatient/outpatient clinic lists and laboratory databases, notifications by local primary care/specialist physicians and allied health services including diabetes education, dietetics and podiatry, advertisements in pharmacies and local media, and word of mouth, as well as third-party mail-outs through the Australian National Diabetes Supply Scheme and National Diabetes Register. An Aboriginal health worker $(\mathrm{KH})$ was employed to identify and recruit indigenous patients.

Of 4639 diabetic patients identified between 2008 and 2011 in the local population of 157,000 (crude diabetes prevalence $3.0 \%), 1668$ (36\%) were recruited to FDS Phase II. Of 2048 self-identified Aboriginal people living in the study area [14], 238 had diabetes (crude prevalence 11.6\%) of whom 113 (47.5\%) were recruited. One hundred and seven (94.7\%) had clinically-defined type 2 diabetes compared with 1509 $(90.5 \%)$ of the total cohort residing within the study area. There were 793 type 2 AC subjects recruited from the local population $(52.6 \%$ of the FDS Phase II type 2 diabetes patients).

\subsection{Demographic and diabetes-specific data collection}

Each FDS Phase II participant was assessed at baseline and invited to attend biennial reviews with questionnaire followup in alternate years. Since longitudinal data collection is at an early stage, we included only baseline data in the present analyses. All face-to-face assessments comprise a comprehensive questionnaire, physical examination and standard fasting biochemical tests [13]. Diabetes type was assessed from diabetes treatment history, body mass index (BMI), age at diagnosis, nature of first presentation, and/or self-identification. Non-insulin treated patients and those $\geq 60$ years of age at diagnosis were usually considered to have type 2 diabetes, as were patients $<60$ years of age at diagnosis and taking insulin at the time of study entry but whose first treatment was not insulin. In these and other cases, case records were consulted for evidence of ketonaemia, as well as islet cell antibody (ICA), GAD antibodies, serum insulin and C-peptide levels, if available.

Ethnic/racial background was based on self-selection, country/countries of birth and parents'/grandparents' birth, and language(s) spoken at home. In line with Australian legal rulings and a range of other studies of Aboriginal Australians 
with diabetes [15-18] we used self-identification and acceptance by the local community as the primary criteria for Aboriginality [19]. No participants identified themselves as from a Torres Strait Islander racial background. Smoking history was categorised as current, ex- or never, and alcohol consumption was assessed as number of standard drinks per day (where one standard drink is equivalent to $10 \mathrm{~g}$ alcohol). Five-year cardiovascular risk was estimated for each patient using a previously validated equation based on FDS Phase I longitudinal data [20].

\subsection{Ascertainment of depression}

Details of all current medications including anti-depressant therapy were documented, including date of commencement. Current depression was assessed using the 9-item Patient Health Questionnaire (PHQ-9), a brief self-assessment of depressive symptoms that is valid and reliable with high sensitivity and specificity in both primary care and in community studies of diabetes [21-23]. Participants rate nine depressive symptoms and their frequency/persistence over the previous 2 weeks. The PHQ- 9 was used to assess major and minor depression syndromes based on Diagnostic and Statistical for Mental Disorders (DSM-IV) criteria. Major depression requires the patient to have, for at least 2 weeks, five or more depressive symptoms for more than half the days, with at least one symptom being either depressed mood or anhedonia. For minor depression, patients should have two to four symptoms for more than half of the days with one of the symptoms being either depressed mood or anhedonia. A recent study in several Aboriginal language groups in central Australia identified the PHQ-9 as the most acceptable and culturally valid of a number of depression screening tools evaluated [24]. The PHQ-9 has also been utilised validly in previous psychometric studies of Indigenous Australians [2527].

A lifetime history of depression was assessed with the Brief Lifetime Depression Scale (BLDS). This instrument was developed and validated by our group for use in diabetes. The BLDS was modelled on the PHQ-9 and included similar items, general format and language, but the participants were asked whether they had ever had a period during their lives lasting for 2 weeks or more when they experienced any of the nine symptoms of depression listed in the DSM-IV/5 [28]. Depression was classified using DSM-IV criteria and was considered to have been present if patients had experienced at least five depression symptoms including depressed mood and/or anhedonia. The year in which the episode(s) occurred was also recorded, along with duration and treatment.

\subsection{Statistical analysis}

Data are presented as proportions, mean $\pm \mathrm{SD}$, geometric mean (SD range), or, in the case of variables which did not conform to a normal or log-normal distribution, median [interquartile range, IQR]. For independent samples, two-way comparisons for proportions were by Fisher's exact test, for normally distributed variables by Student's t-test, and for nonnormally distributed variables by Mann-Whitney U-test. Since age, sex, education level and marital status distributions differed between the Aboriginal and AC groups, generalised linear modelling (GLM) with adjustment for age, sex, education level and marital status was used to determine whether depression and related variables were different between groups. Likewise, GLM with adjustment for age and sex was used to determine whether self-care behaviours including alcohol use, smoking and self-monitoring of blood glucose, and cardio-metabolic risk factors, were different between the Aboriginal and AC groups. Multiple logistic regression (forward conditional variable selection with $P<0.05$ for entry and $P>0.10$ for removal) was used to investigate independent associates of any current depression. A two-tailed significance level of $P<0.05$ was used throughout.

\section{Results}

\subsection{Sample characteristics}

The socio-demographic, anthropometric and diabetes-related characteristics of the two patient groups are summarised in Table 1. The Aboriginal patients were significantly younger both at study entry and diabetes diagnosis, and more likely to be female, than those in the AC group $(P \leq 0.002)$, but they had a similar diabetes duration $(P=0.18)$. They were also less likely to be educated beyond primary level and to be married or in a de facto relationship, but were more likely to smoke $(P \leq 0.006)$. Alcohol intake, BMI and central obesity were similar in the two groups $(P \geq 0.28)$.

\subsection{Prevalence of depression}

Fifty-two of the 107 Aboriginal patients (48.6\%) completed the PHQ-9 questionnaire. The 55 who did not complete the PHQ-9 were of similar age $(53.4 \pm 12.9$ vs $55.2 \pm 10.7$ years, $P=0.44)$, and sex $(32.7 \%$ vs $36.5 \%$ male, $P=0.69)$ to those who did, and they had a similar diabetes duration (10.0 [3.0-19.5] vs 7.7 [3.015.0] years, $P=0.49)$, BMI $\left(31.0 \pm 7.0\right.$ vs $33.0 \pm 7.4 \mathrm{~kg} / \mathrm{m}^{2}$, $P=0.15)$ and $\mathrm{HbA}_{1 \mathrm{c}}(9.3[6.8-11.0] \%$ vs 7.9 [6.3-10.2]\% or 78 [51-97] vs 63 [45-88] $\mathrm{mmol} / \mathrm{mol}, P=0.13)$. There were similarly no between-response group differences in marital status (43.6\% vs $34.6 \%$ currently married/de facto relationship, $P=0.43)$ or educational attainment $(81.1 \%$ vs $87.2 \%$ beyond primary level, $P=0.55$ ), and similar proportions were taking antidepressant medications $(9.1 \%$ vs $9.6 \%, P>0.99)$. Twentyfive of those did not complete the PHQ- 9 (45.5\%) answered the BLDS questions and $68.0 \%$ screened positive for lifetime depression, a higher proportion than the $41.3 \%$ of those who also completed the PHQ-9 $(P=0.047)$. Twenty-one AC patients $(2.6 \%)$ did not complete the PHQ-9. Compared with the 772 who did, they were older $(73.1 \pm 10.0$ vs $67.1 \pm 10.6$ years, $P=0.010)$ and were more likely to be taking antidepressant medications (42.9\% vs $16.8 \%, P=0.005)$, but there were no statistically significant between-group differences in sex, diabetes duration, $\mathrm{BMI}, \mathrm{HbA}_{1 \mathrm{c}}$, marital status, educational attainment or lifetime depression ( $P \geq 0.10$ in each case).

The proportions of Aboriginal and AC patients with different categories of depression are detailed in Table 2. Amongst those completing the PHQ-9, Aboriginal respondents were more than twice as likely to have any current depression 
Table 1 - Baseline demographic and anthropometric variables in Aboriginal and Anglo-Celt participants with type 2 diabetes in The Fremantle Diabetes Study Phase II.

\begin{tabular}{|c|c|c|c|}
\hline & Aboriginal & Anglo-Celt & P-value \\
\hline Number & 107 & 793 & \\
\hline Age (years) & $54.3 \pm 11.8$ & $67.2 \pm 10.6$ & $<0.001$ \\
\hline Sex (\% male) & 34.6 & 50.9 & 0.002 \\
\hline Age at diabetes diagnosis (years) & $43.6 \pm 14.3$ & $57.7 \pm 11.3$ & $<0.001$ \\
\hline Duration of diabetes (years) & $8.0[3.0-16.8]$ & $8.0[2.2-15.0]$ & 0.18 \\
\hline Education beyond primary level (\%) & 84.5 & 93.6 & 0.006 \\
\hline Paid employment (\%) & 22.0 & 29.0 & 0.18 \\
\hline Married/de facto relationship (\%) & 39.3 & 61.7 & $<0.001$ \\
\hline Alcohol use (standard drinks/day) & $0.1[0-1.2]$ & $0.1[0-1.2]$ & 0.58 \\
\hline \multicolumn{4}{|l|}{ Smoking status (\%) } \\
\hline Never & 23.8 & 45.4 & \multirow{3}{*}{$<0.001$} \\
\hline Ex- & 31.7 & 46.5 & \\
\hline Current & 44.6 & 8.1 & \\
\hline Body mass index $\left(\mathrm{kg} / \mathrm{m}^{2}\right)$ & $32.0 \pm 7.3$ & $31.3 \pm 6.2$ & 0.36 \\
\hline Obese by waist circumference $^{\mathrm{a}}(\%)$ & 77.8 & 72.0 & 0.28 \\
\hline
\end{tabular}

Table 2 - Depression and related variables in Aboriginal and Anglo-Celt participants with type 2 diabetes who completed the PHQ-9 questionnaire.

\begin{tabular}{|c|c|c|c|}
\hline & Aboriginal & Anglo-Celt & P-Value \\
\hline Any current depression defined by PHQ-9 (\% (n/N)) & $25.0(13 / 52)$ & $10.6(82 / 772)$ & 0.16 \\
\hline Major current depression defined by PHQ-9 (\% (n/N)) & $15.4(8 / 52)$ & $4.1(32 / 772)$ & 0.029 \\
\hline Lifetime depression defined by BLDS $(\%(n / N))$ & $41.3(19 / 46)$ & $34.7(262 / 755)$ & 0.19 \\
\hline Taking antidepressant medication $(\%(\mathrm{n} / \mathrm{N}))$ & $9.6(5 / 52)$ & $16.8(130 / 772)$ & 0.16 \\
\hline Any current depression and/or on antidepressants $(\%(\mathrm{n} / \mathrm{N}))$ & $30.8(16 / 52)$ & $24.0(185 / 772)$ & 0.81 \\
\hline Controlled depression ${ }^{a}(\%(\mathrm{n} / \mathrm{N}))$ & $18.8(3 / 16)$ & $55.7(103 / 185)$ & 0.10 \\
\hline Untreated depression $(\%(\mathrm{n} / \mathrm{N}))$ & $68.8(11 / 16)$ & $29.7(55 / 185)$ & 0.032 \\
\hline
\end{tabular}

as the ACs, but this difference was attenuated after adjustment for confounding variables (age, sex, marital status and education; $P=0.16$ ). There was an approximately four-fold increase in current major depression in the Aboriginal patients which remained significant after adjustment $(P=0.029)$. Lifetime depression was also more common in the indigenous group, with $41.3 \%$ of patients affected compared with just over one third of the AC, but this difference was not significant after adjustment $(P=0.19)$. The prevalence of depression was $30.8 \%$ for the Aboriginal and $24.0 \%$ for AC participants when those treated with antidepressant medication were combined with those with current depression who were untreated (adjusted $P=0.29$ ). Untreated depression was significantly more common in the Aboriginal patients (adjusted $P=0.032$ ).

\subsection{Associations between any current depression and} sociodemographic variables, self-care behaviours and cardiometabolic risk factors

These data are summarised by racial group in Table 3 . When the two groups were combined, any current depression was associated bivariately with younger age both at study entry and at diagnosis of diabetes, lower educational attainment, smoking status, and increased BMI, fasting serum glucose,
$\mathrm{HbA}_{1 \mathrm{c}}$, and serum triglycerides ( $\mathrm{P} \leq 0.038$ in each case). There was a significant association between increased alcohol intake and any current depression in the Aboriginal group $(P=0.038)$ which persisted after adjustment for age and sex, and which was not seen when the two racial groups were combined. The ACs with any current depression were younger at diagnosis of diabetes, had lower educational attainment and were more likely to smoke, but consumed less alcohol and had a higher BMI and fasting serum glucose than those who were not depressed $(P \leq 0.016$ in each case). Anglo-Celts in paid employment were less likely to have any current depression, but this was of borderline significance $(P=0.052)$. After adjustment for age and sex, significant interactions between any current depression and Aboriginal status were observed for alcohol consumption, educational attainment and paid employment $(P \leq 0.048$ in each case).

\subsection{Independent associates of any current depression}

In multiple logistic regression of the two racial groups combined, education beyond primary level, previous smoking, current smoking, BMI and fasting serum glucose were independently associated with any current depression (see Table 4), but Aboriginal status was not (odds ratio $(95 \%$ confidence interval) 1.65 (0.74-3.65)). In analyses of the 
Table 3 - Impact of any current depression ${ }^{\mathrm{a}}$ on sociodemographic characteristics, self-care behaviours and cardio-metabolic risk factors.

Number
Age (years)

\section{Age (years)}

Sex (\% male)

Education beyond primary level (\%)

Paid employment (\%)

Married/de facto relationship (\%)

Smoking status (never/ex-/current; \%)

Alcohol consumption (standard drinks/day)

Age at diabetes diagnosis (years)

Diabetes duration (years)

Self-monitoring blood glucose (\%)

Body mass index $\left(\mathrm{kg} / \mathrm{m}^{2}\right)$

Supine systolic blood pressure $(\mathrm{mmHg})$

Supine diastolic blood pressure $(\mathrm{mmHg}$

Fasting serum glucose $(\mathrm{mmol} / \mathrm{L})$

$\mathrm{HbA}_{1 \mathrm{c}}$ (\%)

$\mathrm{HbA}_{1 \mathrm{c}}(\mathrm{mmol} / \mathrm{mol})$

Total serum cholesterol $(\mathrm{mmol} / \mathrm{L})$

Serum HDL-cholesterol $(\mathrm{mmol} / \mathrm{L})$

Serum LDL-cholesterol $(\mathrm{mmol} / \mathrm{L})$

Serum triglycerides $(\mathrm{mmol} / \mathrm{L})$

Urinary albumin:creatinine $(\mathrm{mg} / \mathrm{mmol})$

5-year cardiovascular risk (\%)

${ }^{a}$ Any current depression by PHQ-9.

Test for the interaction between Aboriginal status and any current depression after adjusting for age and sex using generalised linear modelling.
Anglo-Celt

P-Value

\begin{tabular}{|c|c|c|c|c|c|c|c|c|c|}
\hline $\begin{array}{l}\text { Any current } \\
\text { depression }\end{array}$ & $\begin{array}{l}\text { No current } \\
\text { depression }\end{array}$ & P-Value & $\begin{array}{l}\text { Any current } \\
\text { depression }\end{array}$ & $\begin{array}{l}\text { No current } \\
\text { depression }\end{array}$ & $P$-Value & $\begin{array}{l}\text { Any current } \\
\text { depression }\end{array}$ & $\begin{array}{l}\text { No current } \\
\text { depression }\end{array}$ & P-Value & \\
\hline 95 & 729 & & 13 & 39 & & 82 & 690 & & \\
\hline $63.9 \pm 11.3$ & $66.6 \pm 10.9$ & 0.024 & $52.7 \pm 5.3$ & $56.0 \pm 11.9$ & 0.17 & $65.7 \pm 10.9$ & $67.2 \pm 10.5$ & 0.22 & 0.42 \\
\hline 48.4 & 50.8 & 0.74 & 23.1 & 41.0 & 0.33 & 52.4 & 51.3 & 0.91 & 0.24 \\
\hline 87.5 & 94.0 & 0.038 & 100.0 & 83.3 & 0.31 & 85.7 & 94.6 & 0.006 & $<0.001$ \\
\hline 21.7 & 30.0 & 0.11 & 33.3 & 16.7 & 0.24 & 20.0 & 30.7 & 0.052 & 0.048 \\
\hline 51.6 & 61.5 & 0.074 & 23.1 & 38.5 & 0.50 & 56.1 & 62.8 & 0.28 & 0.64 \\
\hline $32.6 / 48.4 / 18.9$ & $46.2 / 45.5 / 8.3$ & 0.002 & $30.8 / 23.1 / 46.2$ & $32.4 / 32.4 / 35.1$ & 0.79 & $32.9 / 52.4 / 14.6$ & $46.9 / 46.2 / 6.8$ & 0.009 & $>0.62$ \\
\hline $0.1[0-1.3]$ & $0.1[0-1.2]$ & 0.16 & $1.5[0.2-4.0]$ & 0 [0-0.7] & 0.016 & $0.1[0-0.3]$ & $0.1[0-1.2]$ & 0.010 & 0.038 \\
\hline $53.3 \pm 11.6$ & $57.3 \pm 11.7$ & 0.002 & $43.9 \pm 7.5$ & $45.9 \pm 14.0$ & 0.51 & $54.8 \pm 11.5$ & $57.9 \pm 11.2$ & 0.016 & 0.35 \\
\hline $10.0[4.0-15.0]$ & $8.0[2.0-15.0]$ & 0.12 & $7.0[2.4-12.0]$ & 8.0 [3.0-16.1] & 0.55 & $10.3[4.0-15.6]$ & 8.0 [2.0-14.9] & 0.07 & 0.44 \\
\hline 78.7 & 84.9 & 0.13 & 54.5 & 61.1 & 0.74 & 82.1 & 86.2 & 0.31 & 1.00 \\
\hline $34.0 \pm 7.3$ & $31.1 \pm 6.1$ & $<0.001$ & $34.8 \pm 8.4$ & $32.4 \pm 7.1$ & 0.32 & $33.9 \pm 7.2$ & $31.1 \pm 6.0$ & $<0.001$ & 0.80 \\
\hline $145 \pm 23$ & $146 \pm 22$ & 0.94 & $139 \pm 30$ & $141 \pm 29$ & 0.84 & $146 \pm 22$ & $146 \pm 22$ & 0.82 & 0.97 \\
\hline $81 \pm 13$ & $79 \pm 12$ & 0.23 & $81 \pm 14$ & $79 \pm 16$ & 0.74 & $81 \pm 13$ & $79 \pm 12$ & 0.26 & 0.87 \\
\hline $8.0[6.2-10.2]$ & $7.0[6.2-8.4]$ & 0.001 & $9.2[7.2-13.8]$ & 7.8 [6.3-10.7] & 0.22 & 7.9 [6.2-9.9] & $7.0[6.2-8.4]$ & 0.006 & 0.92 \\
\hline $7.2[6.1-8.3]$ & $6.7[6.2-7.5]$ & 0.019 & $9.7[7.0-11.2]$ & 7.8 [6.3-9.5] & 0.21 & $7.1[6.1-8.0]$ & $6.7[6.2-7.4]$ & 0.11 & 0.54 \\
\hline 55 [43-67] & $50[44-58]$ & & 83 [53-99] & $62[45-80]$ & & $54[43-64]$ & $50[44-57]$ & & \\
\hline $4.4 \pm 1.1$ & $4.3 \pm 1.1$ & 0.61 & $4.7 \pm 1.1$ & $4.6 \pm 1.4$ & 0.84 & $4.3 \pm 1.1$ & $4.3 \pm 1.0$ & 0.85 & 0.94 \\
\hline $1.22 \pm 0.29$ & $1.27 \pm 0.36$ & 0.10 & $1.17 \pm 0.30$ & $1.15 \pm 0.27$ & 0.79 & $1.23 \pm 0.29$ & $1.28 \pm 0.36$ & 0.12 & 0.61 \\
\hline $2.4 \pm 0.9$ & $2.3 \pm 0.9$ & 0.60 & $2.6 \pm 1.0$ & $2.5 \pm 1.2$ & 0.81 & $2.3 \pm 0.9$ & $2.3 \pm 0.9$ & 0.81 & 0.99 \\
\hline $1.7(1.0-2.8)$ & $1.5(0.9-2.4)$ & 0.019 & $2.5(1.5-4.1)$ & $1.9(1.1-3.3)$ & 0.12 & $1.6(1.0-2.6)$ & $1.5(0.9-2.4)$ & 0.16 & 0.46 \\
\hline $3.4(0.9-13.1)$ & $3.0(0.8-10.5)$ & 0.34 & $5.9(1.0-36.2)$ & $5.7(0.8-41.5)$ & 0.94 & $3.1(0.9-10.9)$ & $2.9(0.9-9.6)$ & 0.58 & 0.96 \\
\hline $11.6(4.2-32.1)$ & 10.8 (3.9-29.6) & 0.53 & $15.1(7.4-30.8)$ & $18.2(7.1-46.7)$ & 0.52 & $11.1(3.8-32.0)$ & $10.5(3.8-28.7)$ & 0.65 & 0.96 \\
\hline
\end{tabular}

0.96 
Table 4 - Multiple logistic regression analysis of variables independently associated with any current depression in all patients, and in the Aboriginal and Anglo-Celt groups considered individually. Data are odds ratios and $(95 \%$ confidence intervals).

\begin{tabular}{|c|c|c|c|}
\hline Variable & All & Aboriginal & Anglo-Celt \\
\hline Education beyond primary level & $0.47(0.22-0.995)$ & & $0.37(0.17-0.81)$ \\
\hline Previous smoking & & & $1.76(1.02-3.03)$ \\
\hline Current smoking & $3.92(1.96-7.81)$ & & $3.78(1.73-8.28)$ \\
\hline Body mass index (per $1 \mathrm{~kg} / \mathrm{m}^{2}$ increase) & $1.07(1.03-1.10)$ & & $1.06(1.03-1.10)$ \\
\hline Fasting serum glucose (per $1 \mathrm{mmol} / \mathrm{L}$ increase) & $1.09(1.01-1.18)$ & & $1.10(1.01-1.20)$ \\
\hline Alcohol use (per 1 standard drink increase) & & $1.47(1.001-2.15)$ & \\
\hline
\end{tabular}

separate racial groups, only alcohol was independently associated with any current depression in Aboriginals, while education beyond primary level, previous smoking, current smoking, BMI and fasting serum glucose were independently associated with any current depression in the AC group.

\section{Discussion}

The present study shows that prevalent depression was not significantly increased in urban-dwelling Aboriginal versus AC Australians with type 2 diabetes after adjustment for a range of potentially confounding variables. However, depressive illness was more likely to be major and untreated in the Aboriginal group. Variables associated independently with depression in AC group included educational attainment (inversely), smoking status, BMI and fasting plasma glucose, while alcohol use was the only such associate in the Aboriginal group. In the cohort as a whole, depressed patients were more likely to smoke, to be obese, and to have poor metabolic control. These data have significant implications for the assessment and treatment of patients with type 2 diabetes in Australian communities, and may mirror the situation in other countries with marginalised indigenous populations.

The unadjusted prevalence of depression, whether total or major, in our Aboriginal patients was more than double that in the majority AC ethnic group. This is greater than the equivalent relative prevalence in the general population [10], suggesting that type 2 diabetes increases the risk of depression in Aboriginal as well as AC patients. We did not have control groups without diabetes, but the rates of any current (25.0\%) and lifetime (41.3\%) depression in our Aboriginal patients with type 2 diabetes were higher than the $23 \%$ rate of any previously diagnosed depression in an urban adult indigenous sample from the general Australian population [29]. In addition, the $15.4 \%$ prevalence of current major depression was identical to that assessed in the same way (through PHQ-9) in Australian Aboriginal patients with ischaemic heart disease [26], a condition which is also strongly associated with depression in indigenous communities [30]. Previously published studies showing that diabetes does not increase the risk of depression in Aboriginal communities in Australia [11,12,30] and elsewhere [9] have employed different patient selection procedures and/or psychosocial assessment tools to those in the present study, and even the location of the study (urban vs semi-rural or rural/remote) may complicate comparisons [30].
The relative lack of treatment for depression in our Aboriginal patients could reflect primary and hospital-based healthcare that is ineffective in case detection, accurate diagnosis and initiation of antidepressant treatment in indigenous patients, or unattractive because it does not recognise important cultural sensitivities including the provision of appropriate medication advice [31]. Adherence with treatment could be reduced in Aboriginal patients, perhaps reflecting ethnicity-specific differences in disposable income, prioritisation of health behaviours and the understanding of treatment benefits that can occur in disenfranchised minorities [32,33]. However, the potentially serious consequences of untreated depression, including in the specific context of diabetes [4-7], highlight the need for greater awareness of this diagnosis in the management of indigenous patients. In addition, clinical evidence of excessive alcohol consumption should raise the possibility of associated depression in indigenous patients with type 2 diabetes.

The relatively high alcohol consumption, albeit at a median of $<2$ Australian standard drinks ( $<20$ g ethanol) per day, in the Aboriginal patients might be a cause or consequence of depression [34]. In a study from a remote Aboriginal community in the state of Queensland, indigenous patients with diabetes were more likely to abstain from alcohol, but drinkers with diabetes consumed alcohol at levels that were as harmful and hazardous as in indigenous non-diabetic drinkers [35]. Whether depression is a particular risk factor for heavy drinking in Aboriginals with diabetes requires further study, but it is possible that effective antidepressant treatment could reduce alcohol use, especially if ethanol is a form of selfmedication [36]. Other strategies designed to promote community action, including better training and involvement of primary care physicians, may have limited effectiveness in limiting risk-associated consumption by Aboriginal Australians [37].

The relationship between smoking and depression was stronger for Anglo-Celt patients in the present study, but the rate of smoking in the Aboriginal group was substantial (approaching half the sample). Depression was nevertheless strongly associated with smoking, as well as obesity and poor metabolic control, in the two groups combined, consistent with previous studies [4-7]. Analogous to alcohol consumption, glycaemia may be a cause or an effect of depression [6], but improvements in glycaemic control are associated with fewer depressive symptoms [38]. The relative hypertriglyceridemia seen in the depressed patients may reflect a combination of poor blood glucose control and increased alcohol use by the Aboriginal patients. 
There were suggestions in the data that Aboriginal patients with type 2 diabetes in paid employment and with higher educational attainment had an increased risk of depression, the reverse of the trends in the AC group. This might indicate that the responsibility associated with earning income for a typically extended indigenous family contributes to depression. There is likely to be a complex and racially specific interaction between such socio-cultural factors and depression complicating diabetes.

The present study had limitations. The number of Aboriginal participants was small compared to the AngloCelt group and they may not have reflected the local Aboriginal population. However we recruited almost half of those we identified in the study catchment area, a proportion greater than the $36 \%$ for recruitment to FDS Phase II as a whole. The $48.5 \%$ of Aboriginal patients who completed the PHQ- 9 were similar to those who did not in terms of key sociodemographic, anthropometric and diabetes-specific variables. However, we cannot exclude the possibility that those with PHQ-9 data were different in other respects relevant to depression and its determinants. Nevertheless, other Australian studies involving Aboriginal subjects have shown lower recruitment and response rates and/or have involved samples that were likely to have been subject to bias [11,12,29], while the estimates of depression in the present study were consistent with those in other studies of indigenous samples that utilised the PHQ-9 $[26,30]$. The strengths of the study are its community-based nature, overall large sample size, and detailed data collection supporting multivariable analyses.

Given the substantial differences in culture and community between Aboriginal Australians and the non-Aboriginal population, community-specific and culturally appropriate strategies are needed to promote screening for depression in Aboriginal patients with type 2 diabetes [39]. Identification of the potentially large proportion of patients with unrecognised or untreated or under-treated depression should be linked with a detailed assessment of alcohol use, smoking habit and cardiovascular risk factors which are likely to be relatively adverse but modifiable [40]. Management strategies should include time devoted to an explanation of the nature and value of chronic antidepressant therapy, especially as this usually adds to established polypharmacy [31].

\section{Conflict of interest}

None declared.

\section{Acknowledgements}

We thank the patients and FDS staff for their involvement in the study and PathWest Laboratory Medicine at Fremantle Hospital for laboratory tests. The FDS Phase II by the National Health and Medical Research Council (Project Grant 513781). The present Aboriginal sub-study of the FDS was supported by an unrestricted educational grant from Merck Sharp and Dohme Corporation. TMED is supported by a National Health and Medical Research Council of Australia Practitioner Fellowship (572561).

\section{R E F E R E N C E S}

[1] Campayo A, de Jonge P, Roy JF, Saz P, de la Camara C, Quintanilla MA, et al. Depressive disorder and incident diabetes mellitus: the effect of characteristics of depression. Am J Psychiatry 2010;167:580-8.

[2] Mezuk B, Eaton WW, Albrecht S, Golden SH. Depression and type 2 diabetes over the lifespan: a meta-analysis. Diabetes Care 2008;31:2383-90.

[3] Anderson RJ, Freedland KE, Clouse RE, Lustman PJ. The prevalence of comorbid depression in adults with diabetes: a meta-analysis. Diabetes Care 2001;24:1069-78.

[4] Bruce DG, Davis WA, Starkstein SE, Davis TM. A prospective study of depression and mortality in patients with type 2 diabetes: the Fremantle Diabetes Study. Diabetologia 2005;48:2532-9.

[5] de Groot M, Anderson R, Freedland KE, Clouse RE, Lustman PJ. Association of depression and diabetes complications: a meta-analysis. Psychosom Med 2001;63:619-30.

[6] Lustman PJ, Anderson RJ, Freedland KE, de Groot M, Carney RM, Clouse RE. Depression and poor glycemic control: a meta-analytic review of the literature. Diabetes Care 2000;23:934-42.

[7] Schram MT, Baan CA, Pouwer F. Depression and quality of life in patients with diabetes: a systematic review from the European depression in diabetes (EDID) research consortium. Curr Diabetes Rev 2009;5:112-9.

[8] Marmot M. Social determinants of health inequalities. Lancet 2005;365:1099-104.

[9] Daniel M, Rowley KG, Herbert CP, O’Dea K, Green LW. Lipids and psychosocial status in aboriginal persons with and at risk for Type 2 diabetes: implications for tertiary prevention. Patient Educ Couns 2001;43:85-95.

[10] Australian Government Australian Institute of Health and Welfare (AIHW). The health and welfare of Australia's Aboriginal and Torres Strait Islander people, an overview 2011. Canberra: AIHW; 2011.

[11] Si D, Dowden M, Kennedy C, Cox R, O’Donoghue L, Liddle H, et al. Indigenous community care - documented depression in patients with diabetes. Aust Fam Physician 2011;40:331-3.

[12] Schierhout G, Nagel T, Si D, Connors C, Brown A, Bailie R. Do competing demands of physical illness in type 2 diabetes influence depression screening, documentation and management in primary care: a cross-sectional analytic study in Aboriginal and Torres Strait Islander primary health care settings. Int J Ment Health Syst 2013;7:16.

[13] Davis TM, Bruce DG, Davis WA. Cohort profile: the Fremantle Diabetes Study. Int J Epidemiol 2013;42: 412-21.

[14] Australian Bureau of Statistics. 2006 Census. Canberra: Commonwealth Government of Australia.

[15] Landers J, Henderson T, Craig J. Prevalence and associations of cataract in indigenous Australians within central Australia: the Central Australian Ocular Health Study. Clin Experiment Ophthalmol 2010;38:387-92.

[16] McDermott RA, Li M, Campbell SK. Incidence of type 2 diabetes in two indigenous Australian populations: a 6-year follow-up study. Med J Aust 2010;192:562-5.

[17] Minges KE, Zimmet P, Magliano DJ, Dunstan DW, Brown A, Shaw JE. Diabetes prevalence and determinants in indigenous Australian populations: a systematic review. Diabetes Res Clin Pract 2011;93:139-49.

[18] O’Neal DN, Piers LS, Iser DM, Rowley KG, Jenkins AJ, Best JD, et al. Australian Aboriginal people and Torres Strait Islanders have an atherogenic lipid profile that is 
characterised by low HDL-cholesterol level and small LDL particles. Atherosclerosis 2008;201:368-77.

[19] Davis TM, Hunt K, McAullay D, Chubb SA, Sillars BA, Bruce DG, et al. Continuing disparities in cardiovascular risk factors and complications between aboriginal and AngloCelt Australians with type 2 diabetes: the Fremantle Diabetes Study. Diabetes Care 2012;35:2005-11.

[20] Davis WA, Knuiman MW, Davis TM. An Australian cardiovascular risk equation for type 2 diabetes: the Fremantle Diabetes Study. Intern Med J 2010;40:286-92.

[21] Kroenke K, Spitzer RL, Williams JB. The PHQ-9: validity of a brief depression severity measure. J Gen Intern Med 2001;16:606-13.

[22] Roy T, Lloyd CE, Pouwer F, Holt RI, Sartorius N. Screening tools used for measuring depression among people with Type 1 and Type 2 diabetes: a systematic review. Diabet Med 2012;29:164-75.

[23] Katon W, Lyles CR, Parker MM, Karter AJ, Huang ES, Whitmer RA. Association of depression with increased risk of dementia in patients with type 2 diabetes: the Diabetes and Aging Study. Arch Gen Psychiatry 2012;69:410-7

[24] Brown A. Kurunpa [Spirit]: exploring the psychosocial determinants of coronary heart disease among indigenous men in Central Australia.(Ph.D. thesis) Brisbane: School of Population Health; 2009.

[25] Esler DM, Johnston F, Thomas D. The acceptability of a depression screening tool in an urban, Aboriginal community-controlled health service. Aust N Z J Public Health 2007;31:259-63.

[26] Esler D, Johnston F, Thomas D, Davis B. The validity of a depression screening tool modified for use with Aboriginal and Torres Strait Islander people. Aust N Z J Public Health 2008;32:317-21.

[27] Brown AD, Mentha R, Rowley KG, Skinner T, Davy C, O’Dea $\mathrm{K}$. Depression in Aboriginal men in central Australia: adaptation of the Patient Health Questionnaire 9. BMC Psychiatry 2013;13:271.

[28] Bruce DG, Davis WA, Cetrullo V, Starkstein SE, Davis TM. Clinical impact of the temporal relationship between depression and type 2 diabetes: the Fremantle diabetes study phase II. PLOS ONE 2013;8:e81254.
[29] Gubhaju L, McNamara BJ, Banks E, Joshy G, Raphael B, Williamson A, et al. The overall health and risk factor profile of Australian Aboriginal and Torres Strait Islander participants from the 45 and up study. BMC Public Health 2013;13:661.

[30] Brown A, Carrington MJ, McGrady M, Lee G, Zeitz C, Krum $\mathrm{H}$, et al. Cardiometabolic risk and disease in Indigenous Australians: the heart of the heart study. Int J Cardiol 2014;171:377-83.

[31] Swain L, Barclay L. They've given me that many tablets, I'm bushed. I don't know where I'm going: Aboriginal and Torres Strait Islander peoples' experiences with medicines. Aust J Rural Health 2013;21:216-9.

[32] Cooper H. Investigating socio-economic explanations for gender and ethnic inequalities in health. Soc Sci Med 2002;54:693-706.

[33] Lucas A, Murray E, Kinra S. Heath beliefs of UK South Asians related to lifestyle diseases: a review of qualitative literature. J Obes 2013;2013:827674.

[34] Boden JM, Fergusson DM. Alcohol and depression. Addiction 2011;106:906-14.

[35] McCulloch B, McDermott R, Miller G, Leonard D, Elwell M, Muller R. Self-reported diabetes and health behaviors in remote indigenous communities in Northern Queensland, Australia. Diabetes Care 2003;26:397-403.

[36] Dongier M. What are the treatment options for comorbid alcohol abuse and depressive disorders? J Psychiatry Neurosci 2005;30:224.

[37] Shakeshaft A, Doran C, Petrie D, Breen C, Havard A, Abudeen A, et al. The effectiveness of community action in reducing risky alcohol consumption and harm: a cluster randomised controlled trial. PLoS Med 2014;11:e1001617.

[38] Mazze RS, Lucido D, Shamoon H. Psychological and social correlates of glycemic control. Diabetes Care 1984;7:360-6.

[39] Brown A, Scales U, Beever W, Rickards B, Rowley K, O’Dea $\mathrm{K}$. Exploring the expression of depression and distress in aboriginal men in central Australia: a qualitative study. BMC Psychiatry 2012;12:97.

[40] Lucero AA, Lambrick DM, Faulkner JA, Fryer S, Tarrant MA, Poudevigne $\mathrm{M}$, et al. Modifiable cardiovascular disease risk factors among indigenous populations. Adv Prev Med 2014;2014:547018. 